\title{
Infective Endocarditis with Negative Cultures: A Defiant Diagnosis
}

\author{
Cláudia Rocha*, Vera Clérigo, Lígia Fernandes, André Rodrigues, Dora Sargento, \\ Glória Silva \\ Serviço de Medicina III, Hospital Pulido Valente, Centro Hospitalar Lisboa Norte, Lisboa, Portugal \\ Email: sudney@hotmail.com
}

Received 9 July 2014; revised 1 August 2014; accepted 11 August 2014

Copyright (C) 2014 by authors and Scientific Research Publishing Inc.

This work is licensed under the Creative Commons Attribution International License (CC BY).

http://creativecommons.org/licenses/by/4.0/

(c) (i) Open Access

\begin{abstract}
The diagnosis and management of blood culture-negative endocarditis constitute a real clinical challenge and a systemic approach is necessary for a successful outcome. The authors report a case of a female patient aged 26 , with previous clinical history of valve disease and heart failure NYHA class II, with decompensation of co-morbidities associated with fever, productive cough, nonselective anorexia and unquantified weight loss with one month of evolution with negative blood cultures. Transesophageal echocardiography revealed the presence of severe pulmonary hypertension and mitral valve vegetations in the context of positive serology for $Q$ fever. Herein, the authors report a case of blood culture-negative endocarditis and present a brief review on the management of this medical condition. We highlighted the diagnostic difficulties of blood culturenegative endocarditis and subacute clinical presentation, which sometimes present with fever of unknown origin and complaints of deterioration of cardiac function, thus creating a challenging differential diagnosis.
\end{abstract}

\section{Keywords}

Coxiella burnetii, Endocarditis, Blood Culture-Negative Endocarditis

\section{Introduction}

Blood culture-negative endocarditis (BCNE) defines certain or likely endocarditis in which three or more aerobic and anaerobic blood cultures collected over 48 hours persist negative regardless of extended incubation period (superior to one week) [1] [2]. Definitely, it establishes a significant clinical challenge and is frequently connected with higher morbidity and mortality, when compared with blood culture positive endocarditis [3].

\footnotetext{
*Corresponding author.
}

How to cite this paper: Rocha, C., Clérigo, V., Fernandes, L., Rodrigues, A., Sargento, D. and Silva, G. (2014) Infective Endocarditis with Negative Cultures: A Defiant Diagnosis. Case Reports in Clinical Medicine, 3, $460-464$.

http://dx.doi.org/10.4236/crcm.2014.38101 
BCNE comprises $2.5 \%$ to $31 \%$ of all cases of endocarditis [4].

Infective endocarditis (IE), in particular, is a serious disease whose mortality and morbidity have not considerably decreased over the past 30 years [5]-[7], and is potentially fatal if treatment is not appropriate [8].

The epidemiological profile of IE has changed in recent years, especially in industrialized countries. Once considered a disease affecting mostly young adults diagnosed with valvular disease (mainly rheumatic heart disease), in recent years the incidence has increased in the elderly. The incidence is variable, with a record of 3 10 episodes per 100,000 individuals per year [5] [7] [8].

The Duke criteria are based upon clinical, microbiological and echocardio graphic findings providing high specificity and sensitivity for the diagnosis of IE. Despite the fast development of additional means of diagnosis and the introduction of the Duke criteria, the diagnosis of IE is still a challenge for many clinicians [9] [10] [12], especially when cultures are negative, causing a delay in diagnosis and appropriate treatment [5]. This variation may be explained by several factors such as a difference in diagnostic criteria used; specific epidemiological factors (related to the tedious zoonotic agents); previous use of antibiotics; inadequate techniques of blood cultures or involvement of unknown agents [11] [12].

The proper treatment depends upon the identification of the causative agent, whereby in the presence of BCNE becomes necessary to diversify laboratory techniques [8] [11]. Even with all incremental knowledge of the pathogenesis of BCNE, treatment and outcome of BCNE have not improved substantially over the past 30 years making it important to notify and discuss clinical cases of BCNE, particularly those with special context such as the case presented here.

\section{Case Report}

A 28-year-old melanodermic female, single, unemployed, born in Cape Verde, living in Portugal for one month previously to hospital admission, with a clinical history of heart failure (HF) NYHA class II and valvular heart disease (i.e., severe mitral stenos is, moderate aortic stenos is with aortic regurgitation and tricuspid stenosis). She was admitted to our hospital with fever, profuse nocturnal sweating, cough with pinkish mucous sputum, non-selective anorexia and unquantified weight loss with one month of evolution. Physical examination revealed fever (tympanic temperature $39.2^{\circ} \mathrm{C}$ ), normal blood pressure and sinus tachycardia (heart rate $140 \mathrm{bpm}$ ), rapid breathing rate and dehydrated pale skin and mucous membranes. Systolic murmur grade IV/VI, audible throughout theprecordium, hepatosplenomegaly and mild edema of the lower limbs. The investigations yielded the

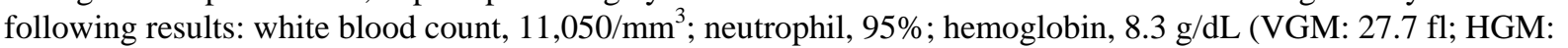
$79.5 \mathrm{pg}$ ), platelets: $150,000 / \mathrm{mm}^{3}$. Blood C-reactive protein $0.7 \mathrm{mg} / \mathrm{L}$. Liver and kidney function tests were in the normal range. Urinalysis and thyroid function was normal. Chest roentogram revealed diffuse and bibasilar interstitial infiltrate.

Assuming a provisional diagnosis of pneumonia, empirical antibiotic therapy with ceftriaxone was started with little improvement. Blood cultures were performed in aerobic and anaerobic conditions; Mycobacteria spp in blood and sputum were negative. Suspicious mitral vegetations on transthoracic echocardiography (TTE) were revealed. A transesophageal echocardiography (TEE) subsequently performed showed severe pulmonary hypertension and vegetations of the mitral (Figure 1) and aortic valves. In the light of this new data, the previous antibiotic was suspended and replaced by ampicillin and gentamicin, with some clinical improvement. Given the negative blood cultures and by reference to housing in formerly rural areas, serology for Coxiella burnetii ( $C$. burnetii) was performed and was positive (IgG titer to phase I and phase II $>1 / 8190$, IgM phase I =1/2048 and IgM phase II = 1/512). The patient started doxycycline (100 mg twice daily) associated with hydroxychloroquine (600 mg once daily) for 21 days with significant clinical and laboratory improvement.

The patient performed a control transthoracic echocardiography at 28 days, which showed clear improvement of pulmonary hypertension, although keeping vegetations of small volume in aortic and mitral valves. She was discharged after one month of hospitalization, continuing with antibiotic therapy with doxycycline and hydroxychloroquine for 18 months and was directed for cardiothoracic surgery consultation.

\section{Discussion}

The diversity of presentation of BCNE constitutes a challenging diagnosis. The clinical history may be considerably variable according to the etiologic agent, absence or presence of previous heart disease and temporal evolution (acute, sub-acute or chronic). The physical examination, despite plainly useful in establishing the diagnostic 


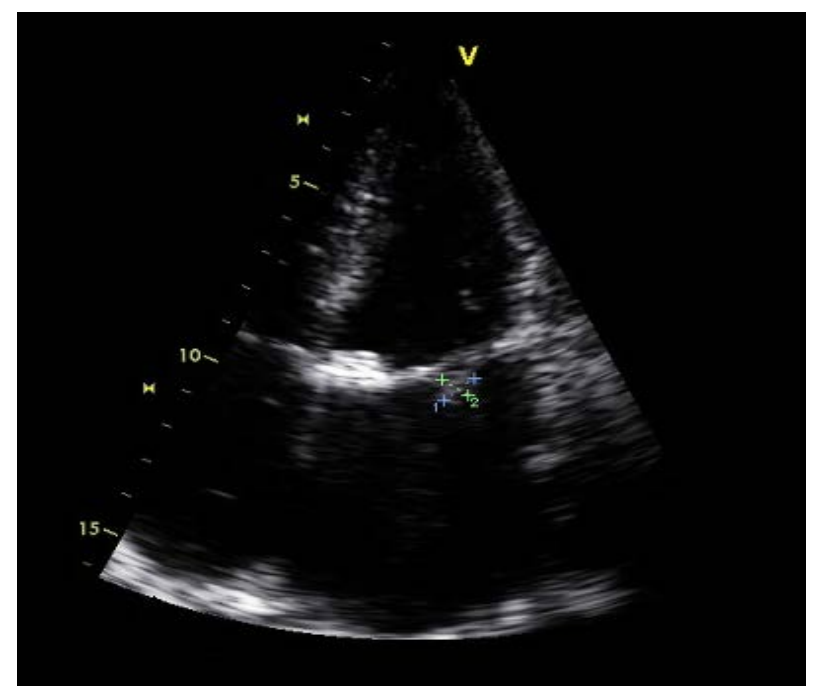

Figure 1. Transoesophageal echocardiography with vegetation in the mitral valve.

hypothesis of endocarditis, it is improbable to aid in defining the etiology [3]. The risk factors for BCNE include previous antibiotic administration, exposure to slow-growing and fastidious non-bacterial microorganisms, underlying valvular heart disease and endocarditis in patients with vascular or intracardiac devices.

Blood cultures are a primordial element in the diagnosis of infective endocarditis [12] and when these come up negative without recognition of any microorganism, several hypotheses should be considered such as the following scenarios enlightened here. Previous administration of antibiotics three days or longer prior to the blood cultures may be responsible for BCNE caused by common and easily growing streptococci and staphylococci, as verified by positive polymerase chain reaction (PCR) identification of DNA from these microorganisms. This situation includes $45 \%$ to $60 \%$ of all cases of culture-negative infective endocarditis (CNIE) [3]. Presence of slow-growing or fastidious organisms requiring specific or enriched media or cell culture systems for intracelular bacteria such as subspecies of streptococci (Abiothrophia spp. which require piridoxal), Gram-negative HACEK group (Haemophilus parainfluenzae, H. aphrophilus, H. paraphrophilus, H. influenzae, Actinobacillusactinomycetemcomitans, Cardiobacteriumhominis, Eikenellacorrodens, Kingellakingae, and K. denitrificans), Brucella spp., Legionella spp. and fungi (Candida, Histoplasma bacilli or Aspergillus). However, recent studies have shown that prolonging incubation up to five days do not increase the isolation of specific microorganisms due to the high likelihood of contamination. To emphasize that although IE caused by anaerobesis uncommon, cultures should be incubated inaerobic and anaerobic environment, to detect any microorganisms such as Bacteroides species or Clostridium spp. Infections by these organisms account for about $40 \%$ to $55 \%$ of cases of CNIE [3]. Presence of intracelular organisms, such as C. burnetii, Bartonella spp., Chlamydia e Tropheryma whipplei (T. whipplei), that requires serologic testing, histologic valvular evaluation or molecular biology to be identified, account for the remaining $5 \%$ of the cases of CNIE. Recently, T. whipplei was the most common isolated organism associated to BCNE.

The Table 1 summarizes some characteristics that increase the degree of suspicion of the referred microorganisms and their additional diagnostic tests to be elaborated.

When the investigation is inconclusive, it should also be suspicious of non-infectious causes of endocarditis classified as non-bacterial thrombotic endocarditis. Given a history of chronic disease or cancer, especially adenocarcinoma associated with disseminated intravascular coagulation, one must consider the hypothesis of marantic endocarditis or aseptic vegetations, complications of systemic lupus erythematosus or anti-phospholipid antibodies syndrome [13]. However, undiagnosed infective endocarditis is probably the most common cause of endocarditis in cancer patients with negative blood cultures [3].

Compared to TTE, TEE shows higher sensitivity in the detection of vegetative lesions, peri-valvular infective foci and murals abscesses with $80 \%$ to $90 \%$ of sensitivity [4].

Regarding the serological methods, they are available for multiple fastidious microorganisms, being included in the Duke criteria for the diagnosis of infective endocarditis, albeit with specific predictive value. Relatively to $C$. 
Table 1. Agents of EICN, suspicious characteristics and complementary means of diagnosis.

\begin{tabular}{|c|c|c|}
\hline Microorganism & Suspicious characteristics & Complementary means of diagnosis \\
\hline Brucella spp. & $\begin{array}{c}\text { Ingestion of animal products derived from } \\
\text { unpasteurized milk } \\
\text { Contact with animals }\end{array}$ & $\begin{array}{l}\text { Blood culture; serology; cultural examination, } \\
\text { immunohistology and surgicalsample PCR }\end{array}$ \\
\hline C. burnetii & Contact with animals & $\begin{array}{c}\text { Serology; cultural examination, immunohistology and } \\
\text { surgicalsample PCR }\end{array}$ \\
\hline Bartonella spp. & $\begin{array}{c}\text { Nutrition and poor sanitary conditions (B. quintana) } \\
\text { Contact with cats (B. henselae) }\end{array}$ & $\begin{array}{l}\text { Blood culture; serology; cultural examination, } \\
\text { immunohistology and surgicalsample PCR }\end{array}$ \\
\hline T. whipplei & Clinical picture with diarrhea & Histology and surgical sample PCR \\
\hline Mycoplasma spp. & & $\begin{array}{c}\text { Serology; cultural examination, immunohistology and } \\
\text { surgicalsample PCR }\end{array}$ \\
\hline Legionella spp. & $\begin{array}{l}\text { Nosocomial infection in patients with } \\
\text { valvular prosthesis }\end{array}$ & $\begin{array}{l}\text { Blood culture; serology; cultural examination, } \\
\text { immunohistologyand surgicalsample PCR }\end{array}$ \\
\hline C. pneumoniae & $\begin{array}{l}\text { Association with aterosclerose and valvular disease; } \\
\text { Cross-reaction with Bartonella spp. }\end{array}$ & $\begin{array}{l}\text { Microimmunofluorescencetest; Serology; cultural } \\
\text { examination, immunohistologyand surgicalsample PCR }\end{array}$ \\
\hline
\end{tabular}

burnetii, a single IgG antibody titer 1:800 is considered major criteria (as seen in our case study). Endocarditis Q fever is characterized by high titers of antigen phase I and II of C. burnetii, and the anti-phase I IgG antibody with a titer of 1:600 is considered to have a high positive predictive value of $98 \%$. Indirect immune of luorescence tests and complementary attachment tests are the most reliable methods for the detection of the etiological agent [3]. The sample indicated for molecular detection for identification of the etiologic agent is excised valvular tissue that is only available in patients undergoing previous resection for valvular replacement [3]. Histopathological examination of the resected valve remains the gold standard test for the diagnosis and is a major criterion in the classification of Duke, with the Gimenez staining used to detection of C. burnetii.

Regarding the treatment of $C$. burnetii BCNE, the combination of doxycycline and hydroxychloroquine (as was established in the patient in question) remains the cornerstone of treatment, confirming a decrease in the duration of antibiotic treatment compared to monotherapy with doxycycline as well as decreased rate of treatment failure or relapse [3]. The surgical valve replacement remains an important option for patients with BCNE, so our patient was referred to cardiothoracic surgery consultation.

\section{Conclusion}

With the case previously described, we highlighted the diagnostic difficulties of BCNE and subacute clinical presentation, which sometimes present with fever of unknown origin and complaints of deterioration of cardiac function, thus creating a differential diagnosis challenge. The fact that the serological diagnosis of fastidious microorganisms may be delayed, it's essential to establish an epidemiological history, as in our case, for a better etiological and therapeutic orientation.

\section{References}

[1] Lepidi, H., Durack, D.T. and Raoult, D. (2002) Diagnostic Methods, Current Best Practices and Guidelines for Histologic Evaluation in Infective Endocarditis. Infectious Disease Clinics of North America, 16, 339-361. http://dx.doi.org/10.1016/S0891-5520(02)00005-3

[2] Durack, D.T., Lukes, A.S. and Bright, D.K. (1994) New Criteria for Diagnosis of Infective Endocarditis: Utilization of Specific Echocardiographic Findings. The American Journal of Medicine, 96, 200-209. http://dx.doi.org/10.1016/0002-9343(94)90143-0

[3] Anthi, K. and Malek, G.M. (2013) Current Issues in the Diagnosis and Management of Blood Culture-Negative Infective and Non-Infective Endocarditis. The Annals of Thoracic Surgery, 95, 1467-1474. http://dx.doi.org/10.1016/j.athoracsur.2012.10.044

[4] Brouqui, P. and Raoult, D. (2001) Endocarditis Due to Rare and Fastidious Bacteria. Clinical Microbiology Reviews, 14, 177-207. http://dx.doi.org/10.1128/CMR.14.1.177-207.2001

[5] European Society of Cardiology Guidelines (2009) Guidelines on the Prevention, Diagnosis, and Treatment of Infective Endocarditis. European Heart Journal, 30, 2369-2413. http://dx.doi.org/10.1093/eurheartj/ehp285 
[6] Herval, L.B., Heloína, L.M.B., Bassoli, C.A., Lena, M.C.V. and José, R.C.J. (2011) Endocarditis with Negative Blood Cultures and Immunological Changes: A Large Challenge. Acta Reumatol Port, 36, 282-286.

[7] Carvalho, M.S., Trabulo, M., Regina, R., Abecasis, J., Leal da Costa, F. and Mendes, M. (2012) A Case of Infective Endocarditis of Native Valve Immunocompromised Patient. Revista Portuguesa de Cardiologia, 31, 35-38. http://dx.doi.org/10.1016/j.repc.2011.10.009

[8] Cordero, A., Escoto, V. and Lopes, L. (2008) Endocardite por Bartonella: Um caso de endocarditecom culturas negativas Bartonellaendocarditis: A Case of Culture-Negative Endocarditis. Revista da Sociedade Portuguesa de Medicina Interna, 15, 186-191.

[9] Maria, W., Rune, A., Lars, O. and Harriet, H. (2003) A Clinical Study of Culture-Negative Endocarditis. Medicine, 82, 263-273. http://dx.doi.org/10.1097/00005792-200307000-00005

[10] Werner, M., Andersson, R., Olaison, L. and Hogevik, H. (2008) A Ten-Year Survey of Blood Culture Negative Endocarditis in Sweden: Aminoglycoside Therapy Is Important for Survival. Scandinavian Journal of Infectious Diseases, 40, 279-285.

[11] Pierre-Edouard, F., Franck, T., Herve, R., Hubert, L., Jean-Paul, C., Jean-Pierre, A., Max, M., Marie, C., Jean-Luc, M., Thierry, C., Frederic, C., Gilbert, H. and Didier, R. (2010) Comprehensive Diagnostic Strategy for Blood CultureNegative Endocarditis: A Prospective Study of 819 New Cases. Clinical Infectious Diseases, 51, 131-140. http://dx.doi.org/10.1086/653675

[12] Werner, M., Fournier, P.E., Andersson, R., Hogevik, H. and Raoult, D. (2003) Bartonella and Coxiella Antibodies in 334 Prospectively Studied Episodes of Infective Endocarditis in Sweden. Scandinavian Journal of Infectious Diseases, 35, 724-727. http://dx.doi.org/10.1080/00365540310015980

[13] Brouqui, P. and Raoult, D. (2001) Endocarditis Due to Rare and Fastidious Bacteria. Clinical Microbiology Reviews, 14, 177-207. http://dx.doi.org/10.1128/CMR.14.1.177-207.2001. 
Scientific Research Publishing (SCIRP) is one of the largest Open Access journal publishers. It is currently publishing more than 200 open access, online, peer-reviewed journals covering a wide range of academic disciplines. SCIRP serves the worldwide academic communities and contributes to the progress and application of science with its publication.

Other selected journals from SCIRP are listed as below. Submit your manuscript to us via either submit@scirp.org or Online Submission Portal.
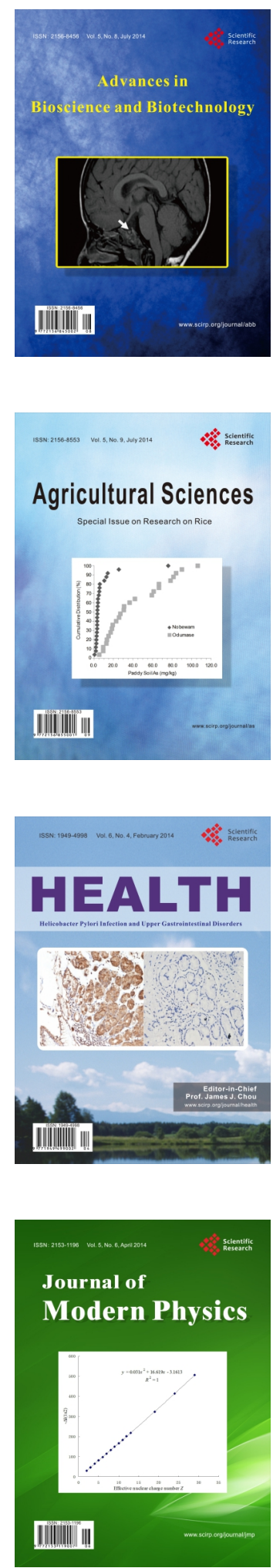
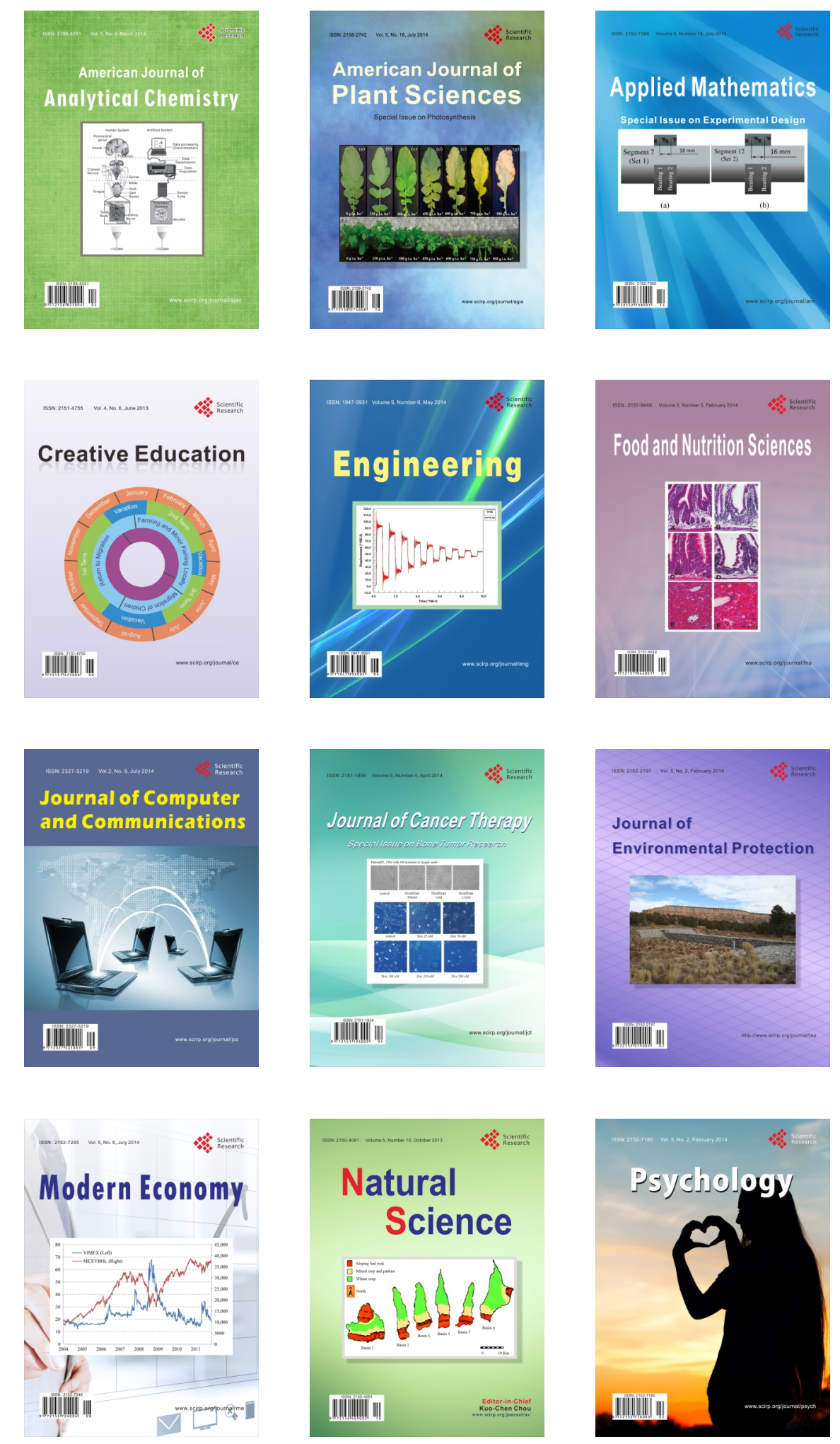